OPEN ACCESS

Edited by:

Jinhang Gao,

Sichuan University, China

Reviewed by:

Huan Tong,

Sichuan University, China

Li YiLing,

The First Affiliated Hospital of China

Medical University, China

*Correspondence:

DongFeng Chen chendf1981@126.com

LiangZhi Wen

wenliangzhi@126.com

${ }^{\dagger}$ These authors have contributed equally to this work

Specialty section:

This article was submitted to

Gastroenterology,

a section of the journal

Frontiers in Medicine

Received: 13 December 2021

Accepted: 19 January 2022

Published: 18 February 2022

Citation:

Wang W, Mu Z, Zhu G, Wang T, Lai S, Guo $Y$, Yin $X$, Wen $L$ and Chen $D$ (2022) A Nomogram for Predicting Portal Hypertensive Gastropathy in

Patients With Liver Cirrhosis: A Retrospective Analysis.

Front. Med. 9:834159.

doi: 10.3389/fmed.2022.834159

\section{A Nomogram for Predicting Portal Hypertensive Gastropathy in Patients With Liver Cirrhosis: A Retrospective Analysis}

WenSheng Wang ${ }^{\dagger}$, ZhiYong Mu ${ }^{\dagger}$, GuangXi Zhu, Tao Wang, ShuJie Lai, Yan Guo, XinRu Yin, LiangZhi Wen* and DongFeng Chen*

Department of Gastroenterology, Daping Hospital, Army Medical University, Chongqing, China

Background: There is an urgent need for non-invasive methods for predicting portal hypertensive gastropathy (PHG). This study aims to develop and validate a non-invasive method based on clinical parameters for predicting $\mathrm{PHG}$ in patients with liver cirrhosis (LC).

Methods: The overall survival (OS) and hepatocellular carcinoma (HCC)-free survival were evaluated in LC patients, both with and without PHG. A prediction model for PHG was then constructed based on a training dataset that contained data on 492 LC patients. The discrimination, calibration, and clinical utility of the predicting nomogram were assessed using the $\mathrm{C}$-index, calibration plot, and decision curve analysis. Internal validation was conducted using a bootstrapping method, and further external validation using data on the 208 other patients.

Results: LC patients with PHG had a worse prognosis compared with those without PHG. A nomogram was constructed using clinical parameters, such as age, hemoglobin content, platelet count and Child-Pugh class. The C-index was 0.773 (95\% Cl: 0.730-0.816) in the training cohort, 0.761 after bootstrapping and 0.745 (95\% Cl: $0.673-$ 0.817 ) in the validation cohort. The AUC values were $0.767,0.724$, and 0.756 in the training, validation and total cohorts, respectively. Well-fitted calibration curves were observed in the training and validation cohorts. Decision curve analysis demonstrated that the nomogram was clinically useful at a threshold of $15 \%$.

Conclusion: The nomogram constructed to predict the risk of developing PHG was found to be clinically viable. Furthermore, $\mathrm{PHG}$ is an independent risk factor for OS of $\mathrm{LC}$, but not for the occurrence of HCC.

Keywords: cirrhosis, portal hypertensive gastropathy, logistic regression, nomogram, predictors

\section{INTRODUCTION}

Portal hypertensive gastropathy (PHG) is a critical but ignored complication of liver cirrhosis (LC), and is frequently diagnosed using endoscopy through the observation of its characteristic mosaic-like pattern with or without red spots. Histologically, PHG is manifested as dilated capillaries or venules in the mucosa or submucosa of the stomach without erosion, inflammation, or fibrinous 
thrombi (1). It can be caused by cirrhotic or non-cirrhotic portal hypertension that result in hyperdynamic circulation and gastric congestion, although its exact pathogenesis is unclear (2). The prevalence of PHG has been reported to greatly vary from about 35 to $80 \%$ in cirrhotic patients due to the use of different classification criteria and study populations (1).

Esophageal varix (EV) is the most common cause of upper gastrointestinal bleeding in cirrhotic patients with portal hypertension. Addition to EV, PHG is also one of the most common causes of cirrhotic related upper gastrointestinal bleeding which should be took into account. The incidence of PHG-related acute and chronic gastrointestinal bleeding is about $2-12 \%$ and $3-60 \%$, respectively (1). Acute bleeding from PHG is less frequent than from varices, but it might be life-threatening as well, and the incidence of bleeding increases as the PHG gets worse. Previous research reported that bleeding-related mortality was up to $12.5 \%$ in the PHG because of the diffuse lesion being difficult for treating $(3,4)$. Moreover, PHG was more prevalent in patients with more fibrosis on liver biopsy (26\% in patients with Ishak score of 3 and $51 \%$ in patients with Ishak score of 6 ), which suggested that PHG could be associated with more severe portal hypertension $(5,6)$.

Although electronic gastroscopy detection (EGD) is the golden standard for the diagnosis of PHG, EGD has not been routinely used for the screening of PHG in patients with cirrhosis due to its invasive procedure, poor tolerance and high cost $(1,7)$. What's worse, the invasive detection may induce serious esophageal and gastric varices rupture and hemorrhage during the EDG procedure. And the endoscopists usually focus on the varices while underestimate the severity of PHG. However, there is absence of suitable non-invasive technique to assess PHG to date $(8-10)$. Therefore, it is urgent to construct a non-invasive method to screen PHG in patients with cirrhosis.
In this study, we aimed to assess the effect of PHG on LC prognosis, explore the relationship between PHG and other decompensated endpoints of LC such as hepatocellular carcinoma (HCC) incidence, and construct a nomogram model to predict $\mathrm{PHG}$.

\section{METHODS \\ Study Patients}

This was a retrospective study, and hospitalized patients with liver cirrhosis were admitted to the Department of Gastroenterology, Daping Hospital of Army Medical University in China, between August 2012 and June 2018. Patients with incomplete data or diagnosis of liver cancer at inclusion were excluded. In summary, 700 cirrhotic patients who had undergone gastroscopy during hospitalization were included in the final dataset. Cirrhosis was diagnosed based on a combination of clinical, laboratory and radiological indices, or histological information. The presence and severity of PHG was evaluated using gastroscopy according to the wide-used McCormack standard: "Mild" with features like fine pink speckling (scarlatina-type rash), and mosaic pattern (snakeskin appearance); "Severe" as discrete red spots or diffuse hemorrhagic lesion (11). The patients were followed up regularly in the outpatient department or through a telephone interview to record survival and hepatocarcinogenesis up to July 2019. The primary endpoint was death occurrence, and the secondary endpoint was incidence of HCC.

The study was approved by the Ethics Committee of Daping Hospital, the Third Affiliated Hospital of Army Medical University. All procedures were performed in accordance with the ethical standards of the institutional research committee.

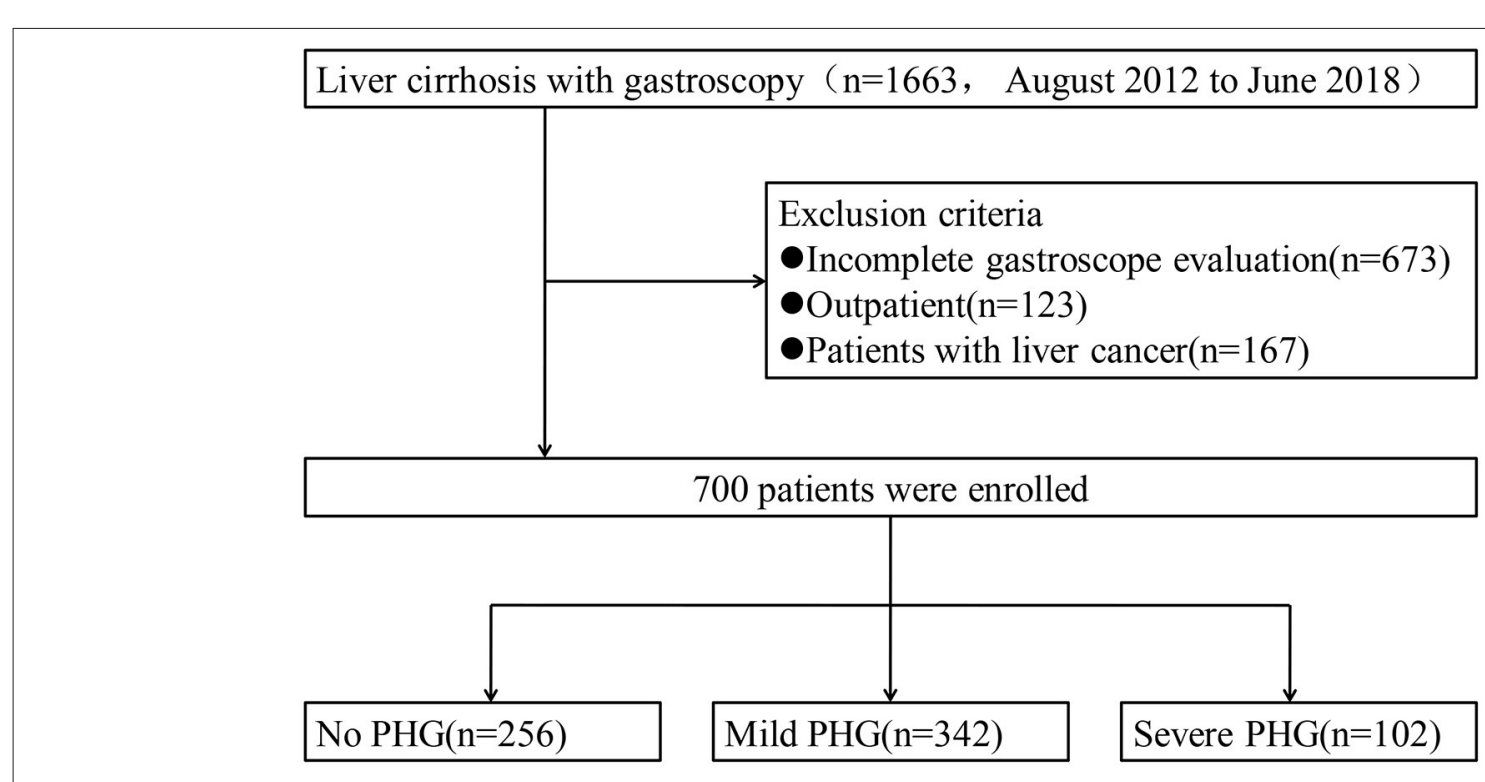

FIGURE 1 | The study flow diagram. 
TABLE 1 | The clinicopathological characteristics of patients enrolled in this study.

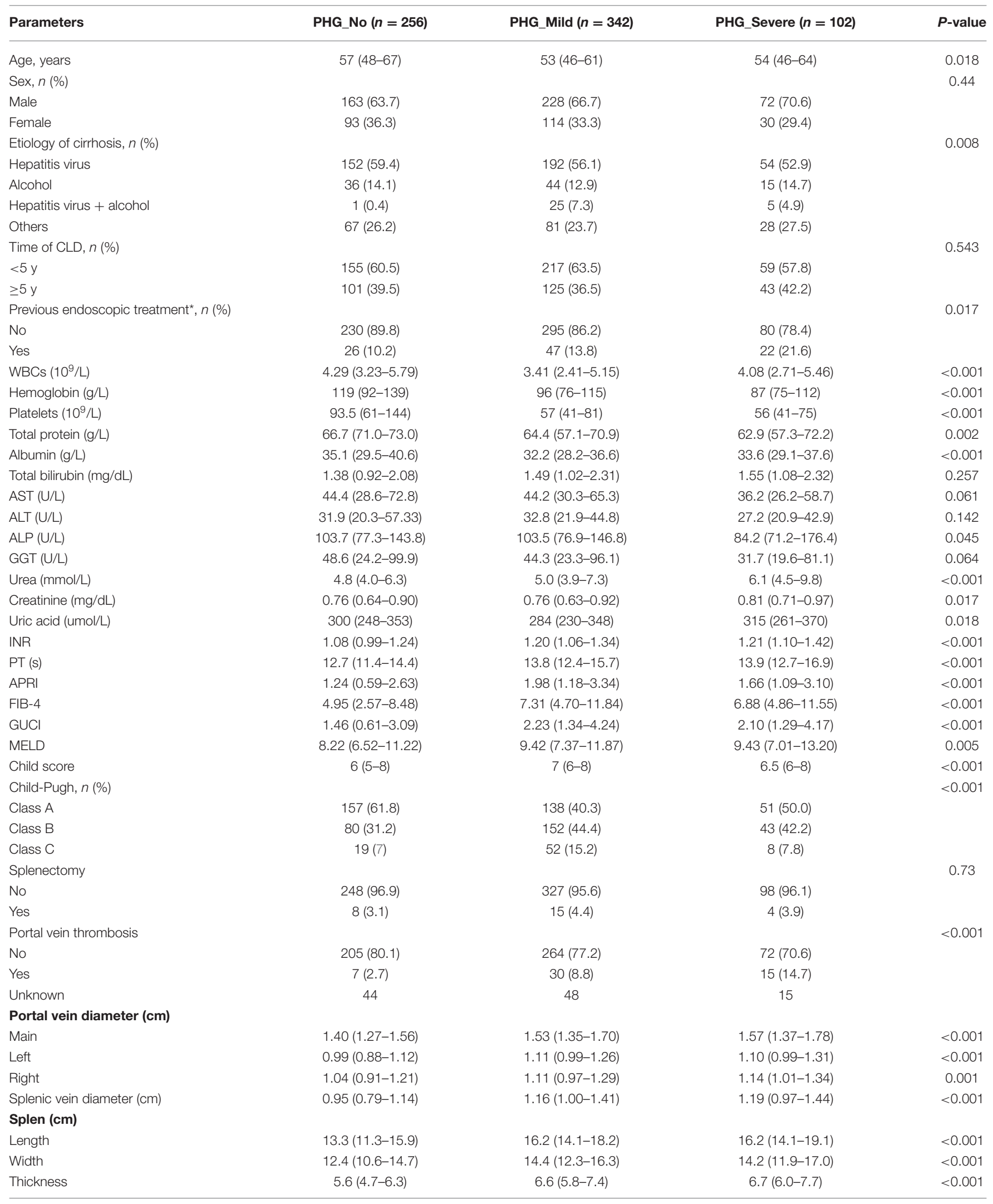

${ }^{*}$ Previous endoscopic treatment means variceal ligation or sclerotherapy ever before. 
TABLE 2 | Cox regression analysis demonstrating the association between variables and OS.

\begin{tabular}{|c|c|c|c|c|c|c|}
\hline \multirow[t]{2}{*}{ Parameters } & \multicolumn{3}{|c|}{ Univariate analysis } & \multicolumn{3}{|c|}{ Multivariate analysis } \\
\hline & HR & $95 \% \mathrm{Cl}$ & $p$-value & HR & $95 \% \mathrm{Cl}$ & $p$-value \\
\hline Age & 1.052 & $1.037-1.066$ & $<0.001$ & 1.061 & $1.045-1.078$ & $<0.001$ \\
\hline Sex & 1.095 & $0.779-1.540$ & 0.600 & NA & & \\
\hline PHG (yes vs. no) & 1.587 & $1.113-2.264$ & 0.011 & 1.587 & $1.097-2.295$ & 0.014 \\
\hline Etiology of cirrhosis & 1.256 & $1.074-1.470$ & 0.004 & 1.066 & $0.899-1.264$ & 0.465 \\
\hline Time of CLD & 0.803 & $0.570-1.132$ & 0.211 & NA & & \\
\hline Previous endoscopic treatment & 1.188 & $0.761-1.856$ & 0.448 & NA & & \\
\hline WBCs $\left(10^{9} / \mathrm{L}\right)$ & 1.072 & $1.020-1.127$ & 0.006 & 1.050 & $0.997-1.106$ & 0.064 \\
\hline Hemoglobin & 0.988 & $0.982-0.993$ & $<0.001$ & 0.987 & $0.980-0.994$ & $<0.001$ \\
\hline Platelets $\left(10^{9} / \mathrm{L}\right)$ & 0.999 & $0.997-1.002$ & 0.621 & NA & & \\
\hline AST & 1.000 & $0.998-1.001$ & 0.715 & NA & & \\
\hline ALT & 0.998 & $0.995-1.001$ & 0.162 & NA & & \\
\hline APRI & 1.035 & $1.013-1.058$ & 0.002 & 1.073 & $0.992-1.161$ & 0.078 \\
\hline FIB-4 & 1.013 & $1.009-1.018$ & $<0.001$ & 1.001 & 0.989-1.013 & 0.848 \\
\hline GUCI & 1.027 & $1.014-1.040$ & $<0.001$ & 0.960 & $0.908-1.015$ & 0.155 \\
\hline MELD & 1.078 & $1.045-1.112$ & $<0.001$ & 1.092 & $1.040-1.147$ & $<0.001$ \\
\hline Child-Pugh class & 1.879 & $1.491-2.369$ & $<0.001$ & 1.314 & $0.992-1.740$ & 0.057 \\
\hline Splenectomy & 0.366 & $0.091-1.476$ & 0.158 & NA & & \\
\hline
\end{tabular}

TABLE 3 | Survival rate based on PHG presence and grade.

\begin{tabular}{|c|c|c|c|c|c|c|c|c|c|c|}
\hline \multirow[t]{2}{*}{ PHG grade } & \multicolumn{2}{|c|}{ Follow-up months } & \multicolumn{2}{|c|}{ Mean survival } & \multirow[b]{2}{*}{ 1-year } & \multirow[b]{2}{*}{$95 \% \mathrm{Cl}$} & \multicolumn{2}{|c|}{ Overall survival rate } & \multirow[b]{2}{*}{ 5-year } & \multirow[b]{2}{*}{$95 \% \mathrm{Cl}$} \\
\hline & Mean \pm SD & Median & Months & $95 \% \mathrm{Cl}$ & & & 3-year & $95 \% \mathrm{Cl}$ & & \\
\hline PHG_No & $35.2 \pm 23.6$ & 35 & 68.2 & $64.4-72.1$ & 0.929 & $0.896-0.963$ & 0.843 & $0.794-0.895$ & 0.763 & $0.697-0.834$ \\
\hline PHG_Yes & $28.1 \pm 23.1$ & 24.5 & 59.0 & $55.8-62.3$ & 0.857 & $0.822-0.893$ & 0.752 & $0.706-0.802$ & 0.652 & 0.589-0.721 \\
\hline PHG_Mild & $28.8 \pm 23.9$ & 25 & 59.8 & $56.1-63.4$ & 0.857 & $0.817-0.898$ & 0.757 & $0.705-0.814$ & 0.666 & $0.597-0.743$ \\
\hline PHG_Severe & $25.9 \pm 20.6$ & 23 & 52.2 & $46.0-58.3$ & 0.855 & $0.786-0.931$ & 0.738 & $0.645-0.845$ & 0.605 & $0.472-0.776$ \\
\hline
\end{tabular}

\section{Clinicopathological Variables}

Baseline data including sex, age, the etiology of LC, and laboratory findings were collected. Child-Pugh classification, APRI [aspartate aminotransferase (AST)-to-platelet ratio index], FIB4 and GUCI (Göteborg University Cirrhosis Index) scores for fibrosis and Model for End-stage Liver disease (MELD) score upon hospitalization were also calculated and extracted for each patient (12).

\section{Statistical Analysis}

All statistical analysis were performed using SPSS (version 23.0) for Windows and $R$ (version 3.5.2) software. The normal distribution of continuous variables was assessed using the Shapiro-Wilks test. Non-normally distributed variables were shown as medians and interquartile ranges (IQRs), while categorical ones were expressed as absolute numbers and percentages. The Kruskal-Wallis test was used to compare the continuous variables, while categorical variables were compared using the chi-square test. Survival and HCC development were analyzed using the Kaplan-Meier method and the results were compared using the log-rank test.
The Cox proportional hazard model was used to identify independent prognostic factors for survival, and hazard ratios (HR) with a $95 \%$ confidence interval (CI) were calculated. The patients were then randomly divided into two cohorts using a 70:30 ratio (13), and the former was used as the training cohort for nomogram construction. Univariate logistic regression was used to identify factors that were significantly associated with PHG, and the odds ratios (OR) with a 95\% CI were calculated. Variables in the univariate logistic regression with a $95 \%$ CI that did not cross 1 and $p<0.05$ were subsequently involved into multivariate logistic regression analysis. The independent risk factors associated with PHG obtained from the multivariate logistic regression were incorporated into the nomogram. A bootstrapping method with 1,000 iterations was used for internal validation $(14,15)$, and external validation was performed using the remaining $30 \%$ of patients. The discriminatory power of the nomogram was expressed in terms of a concordance index (Harrell's C-index) and the area under the curve (AUC) of the receiver operating characteristic (ROC) curve $(16,17)$, with values closer to 1 (range $0.5-1$ ) indicating higher discrimination ability. Calibration plots were drawn to assess the discrepancy between the real and the nomogram-predicted probabilities (18), 

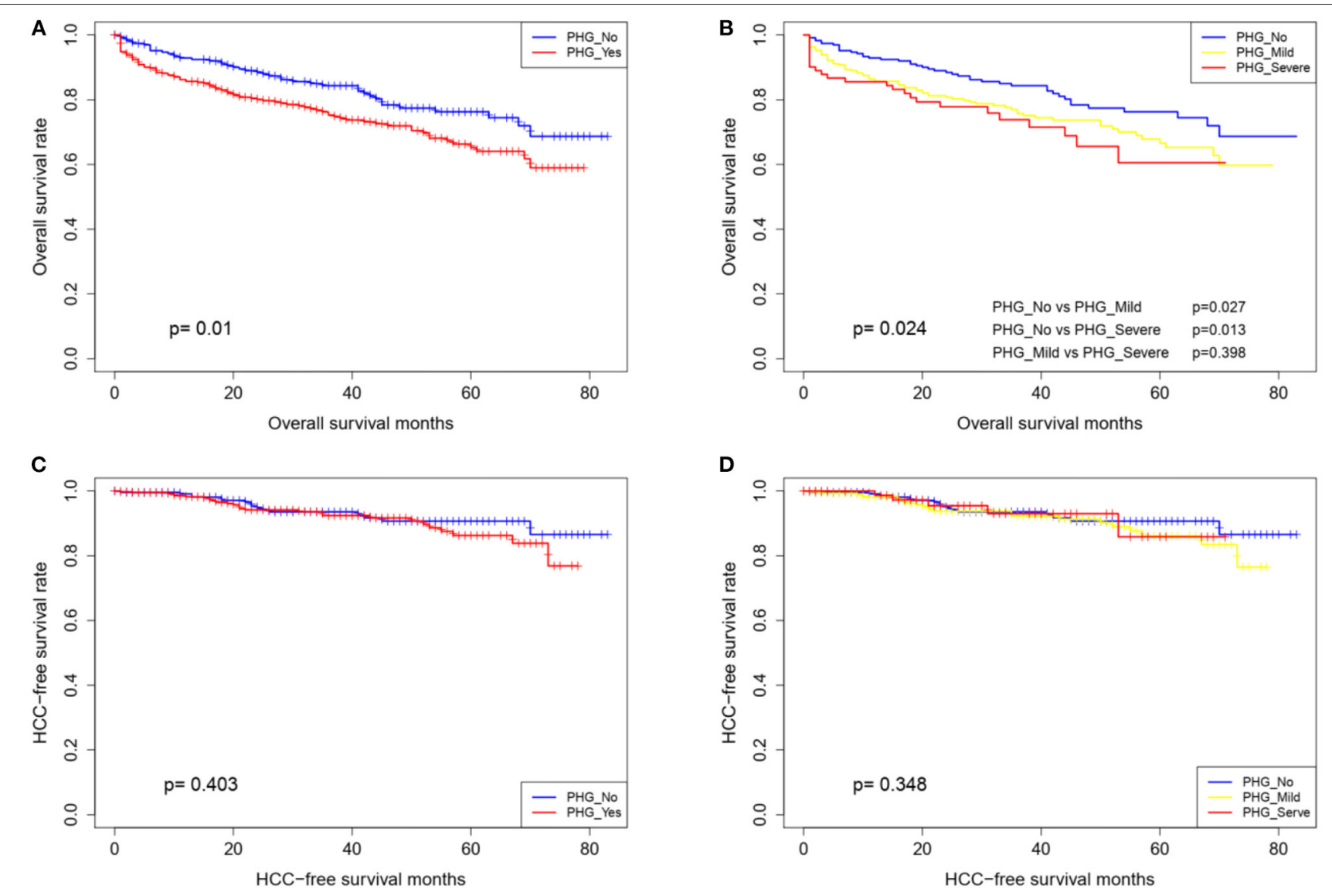

FIGURE 2 | Overall survival and HCC-free survival curves of patients stratified according to the presence $(\mathbf{A}, \mathbf{C})$ and grade of PHG $(\mathbf{B}, \mathbf{D})$.

which were repeated using the validation cohort. Since AUC alone was not enough for decision-making (19), the clinical utility of the nomogram was evaluated through a decision curve analysis (DCA), which quantified the net benefits at different threshold probabilities (20). All tests were two-tailed, and $P$ values of $<0.05$ were considered statistically significant.

\section{RESULTS}

\section{Patient Characteristics}

Of the 1,663 patients with liver cirrhosis who had undergone gastroscopy at our hospital from August 2012 to June 2018, 963 were excluded (Figure 1) and the remaining 700 were enrolled in this study. The clinical characteristics of these patients are summarized (Table 1). PHG was observed in 444 (63.4\%) patients, of which 342 had mild PHG and 102 had severe PHG, and the respective median age of each group was 53 and 54 years old, respectively. Furthermore, two-thirds (66.1\%) of patients were males. Hepatitis virus $(\mathrm{HBV} / \mathrm{HCV})$ infection was the most frequent etiological factor, followed by alcohol abuse. Although $38.4 \%$ of patients had a history of chronic liver disease spanning more than 5 years, fewer than 100 patients had undergone endoscopic therapies, such as ligation or sclerosis for varices.
Child-Pugh classification and MELD scores showed significant differences between the PHG and non-PHG groups, indicating greater severity of LC in the former $(p<0.01)$. In the stratified PHG analysis, multiple laboratory parameters including white blood cell (WBC) and platelet counts were also found to be significantly different, as previously reported $(5,6)$. APRI, FIB4 and GUCI are three non-invasive indices of liver fibrosis and cirrhosis that show perfect diagnostic performance $(12,21)$, and were found to be significantly elevated in the PHG group (Table 1; $p<0.001$ ). This is consistent with the results of a previous study that found an association between PHG and advanced fibrosis (5).

\section{Survival Analysis of Patients With LC}

Eighty patients were lost during the mean follow-up period of 30.7 months. At the end of the follow-up,144 patients had died and 44 had developed HCC. As shown in Table 2, age, PHG, hemoglobin content and MELD scores were found to be associated with poor prognosis, as shown by the univariate and multivariate cox regression analysis. Furthermore, patients with PHG showed worse survival, compared with those without PHG $(\mathrm{HR}=1.587,95 \% \mathrm{CI}: 1.097-2.295, P=0.014)$, with a mean survival duration of 59 months, compared with 68.2 months for 
TABLE 4 | Baseline characteristics of the training and validation cohorts.

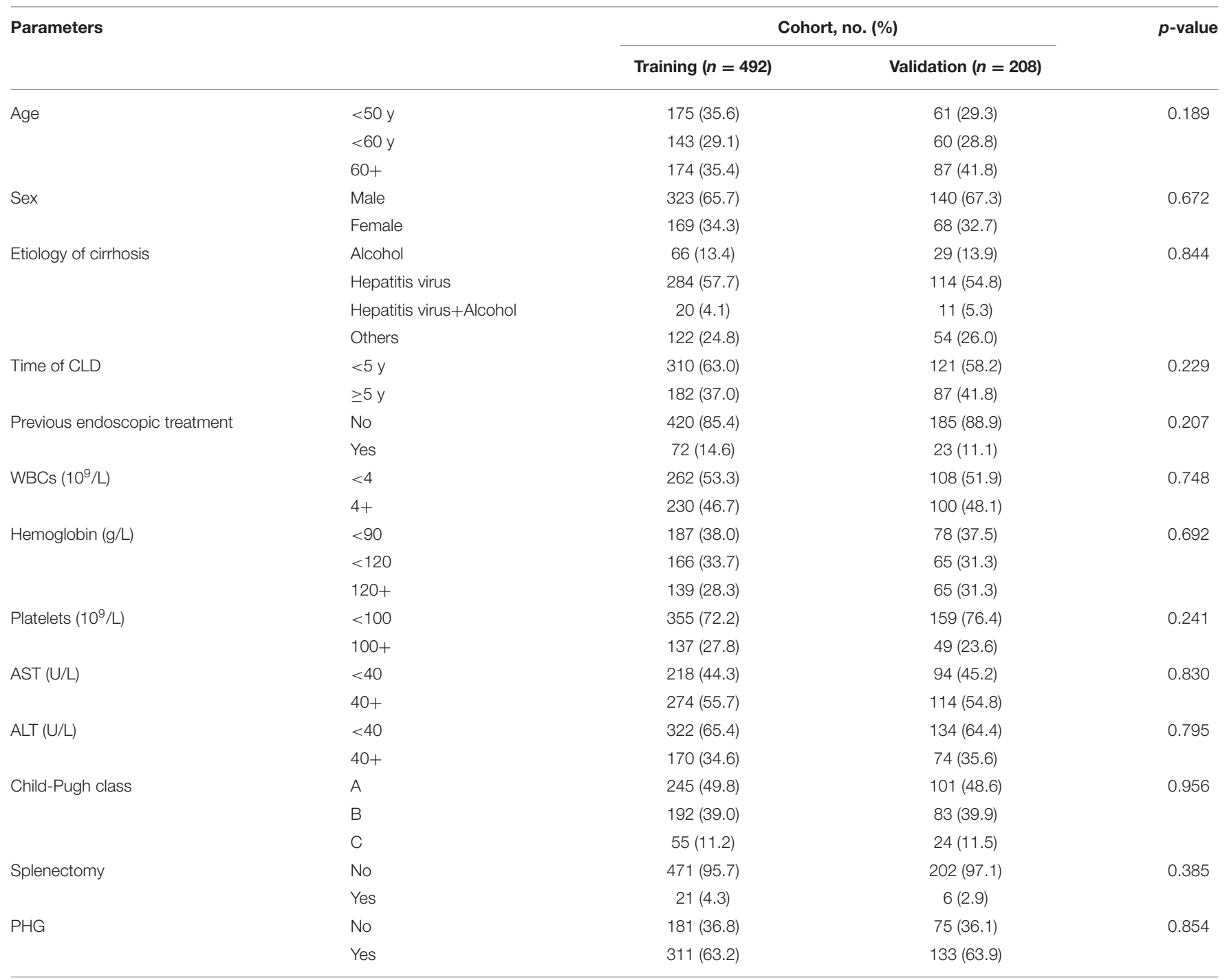

patients without PHG. The survival duration further decreased to 52.2 months in patients with severe PHG. The survival rate of the patients also worsened upon the occurrence and aggravation of PHG (Table 3 and Figure 2), and the 5-year survival rate among those with severe PHG was only $60 \%$. Furthermore, HCC was observed in 16, 23, and 5 patients with no, mild and severe PHG, respectively, during the follow-up period, but was not significantly correlated with the presence or severity of PHG ( $p$ $<0.05$; Figures 2C,D).

\section{Comparison Between the Training and Validation Cohort}

Of the 700 patients, 492 were assigned to the training cohort to be used to construct the nomogram, and 208 were assigned to the validation cohort to be used for external model validation. Based on their clinical significance, the continuous variables were converted into categorical variables. Baseline data between the training and validation cohorts showed no significant differences (Table 4).

\section{Nomogram Development}

The univariate logistic regression analysis of the training group showed that age, WBC count, hemoglobin content, platelet count and Child-Pugh class $(p<0.05)$ were the significant risk factors for PHG. The non-conditional binary multivariate logistic regression analysis of the above revealed that all factors, excluding WBC count were independent risk factors for PHG (Table 5), and were included in the individualized nomogram prediction model (Figure 3A). The predicted risk corresponding to the total score was calculated as the sum of each indicator. The nomogram showed that hemoglobin content was the largest contributor to the risk score, followed by platelet count and Child-Pugh class.

\section{Nomogram Performance}

The nomogram model was further evaluated regarding its discrimination, calibration and clinical utility abilities. The Cindex was 0.773 (95\% CI: $0.730-0.816), 0.761$ and 0.745 (95\% CI: $0.673-0.817)$ in the training cohort, after internal validation 
TABLE 5 | Logistic regression analysis on the training cohort.

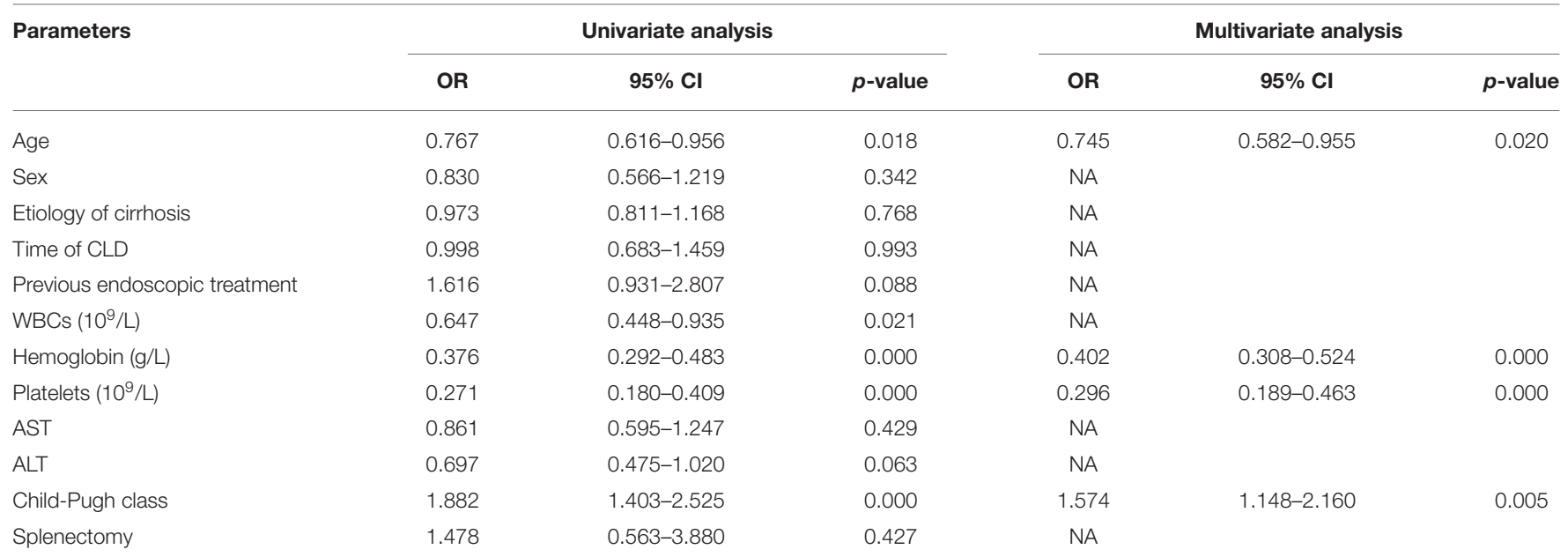

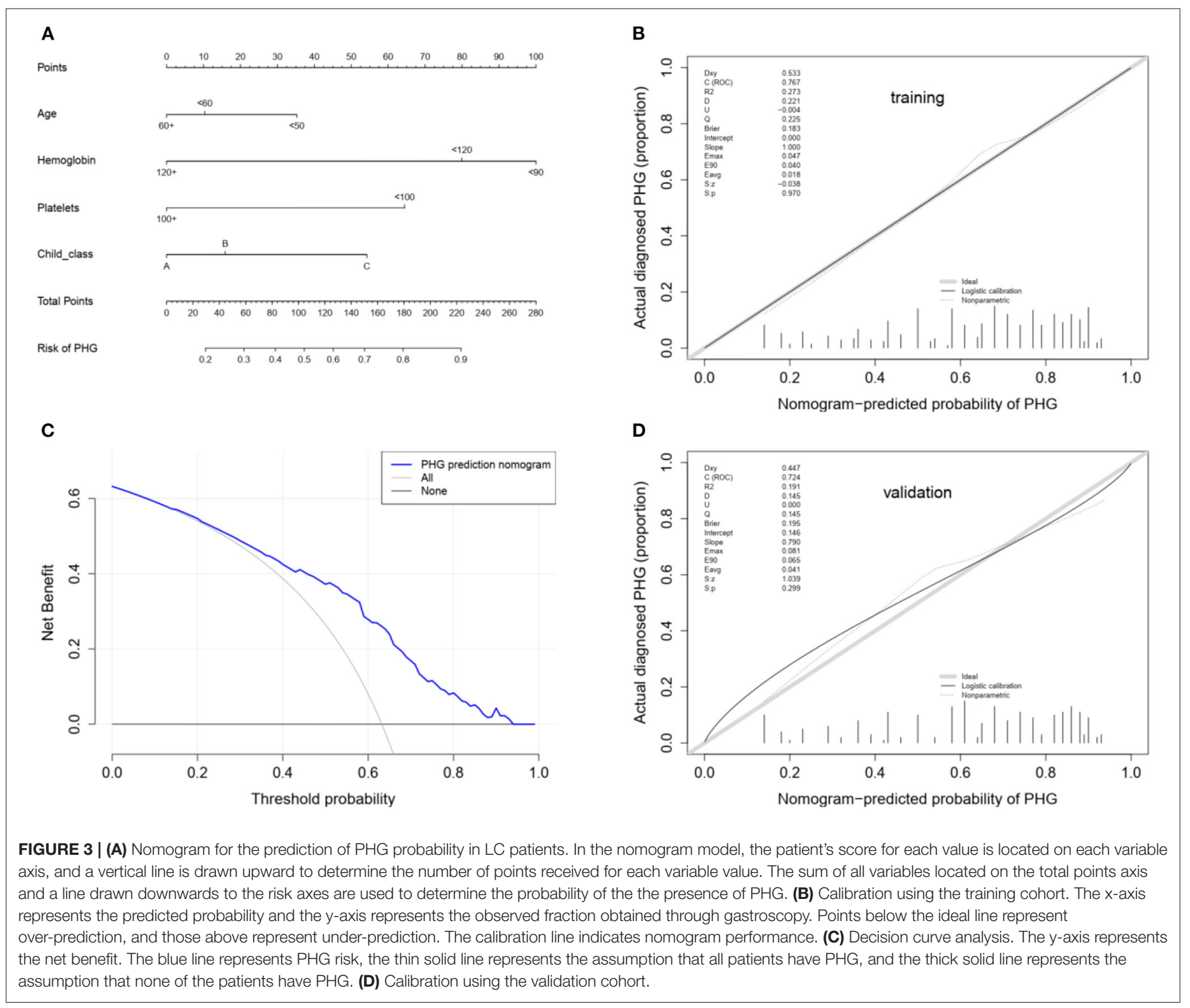




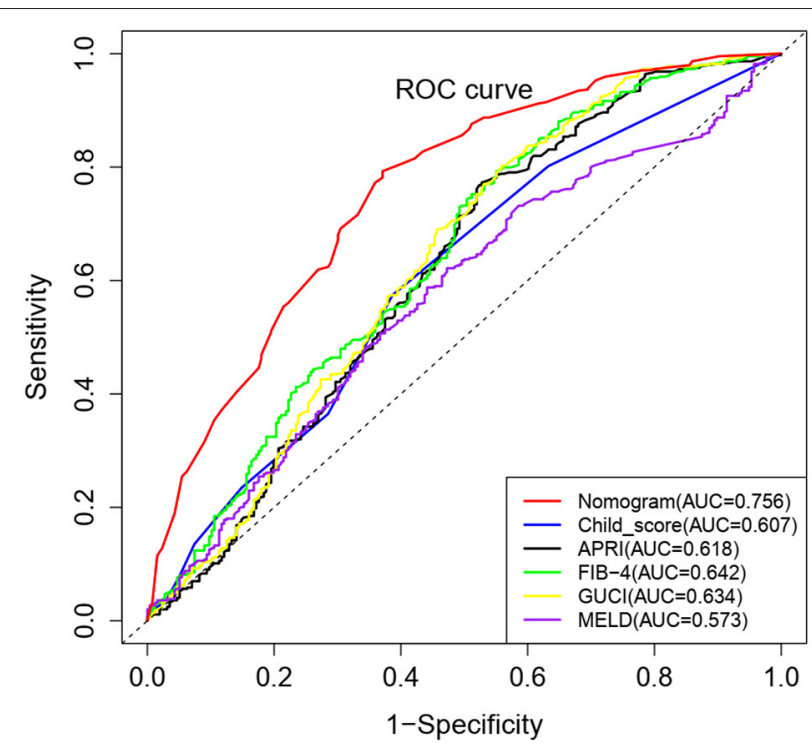

FIGURE 4 | Comparison of ROC curves for different non-invasive parameters using the complete cohort. The nomogram model has the highest diagnostic accuracy with an AUC value 0.756 .

through bootstrapping with 1,000 iterations and in the validation cohort, respectively. Statistically similar C-indices between the training and validation cohorts $(P=0.745)$ indicated that the model was reproducible. The sensitivity, specificity, positive predictive value (PPV), negative predictive value (NPV) and accuracy for the nomogram in the training cohort were 88.7, $48.6,74.8,71.5$, and $74.0 \%$, respectively. Furthermore, the AUCs for the training and validation cohorts were 0.767 and 0.724 , respectively, and that of the entire cohort was 0.756 . The ROC curve was also used to compare the AUC values of other factors including APRI, FIB-4, GUCI, Child-Pugh class, and MELD score. The nomogram model gave the best satisfactory distinction (Figure 4). However, AUC values of $<0.65$ obtained for each indicated that none of these factors can be used to effectively diagnose PHG. The calibration plots for the training and validation groups are shown in Figures 3B,D, and both were similar to the ideal curve. Furthermore, the $P$-values of 0.970 and 0.299 obtained in the calibration test showed that the predictive ability of the nomogram was also close to the actual probability. The decision curves for the training cohort are shown in Figure 3C, and indicated that the model can be used to accurately predict PHG in cirrhotic patients with a threshold probability ranging from 15 to $93 \%$.

\section{DISCUSSION}

Although EGD is the golden standard for the diagnosis of PHG, endoscopic screening is limited owing to its invasive operation, high cost and the need for anesthetic. Thus, noninvasive methods for PHG diagnosis are needed. However, visualized non-invasive model for PHG predicting has not been reported before. In this study, we firstly developed and validated a visualized, non-invasive, clinical parameter-based nomogram for the prediction of PHG in cirrhosis.

Nomograms are widely used in oncology and medicine as prognostic/diagnostic model, and their user-friendly visual interfaces and accurate predictive performance are greatly beneficial for clinical decision making $(22,23)$. To the best of our knowledge, this study was the first to apply a nomogram for the prediction of PHG. We developed and validated a relatively accurate predictive nomogram to be used as a noninvasive diagnostic tool for PHG in LC patients using four easily measurable variables. The prognostic implications of PHG on overall and HCC-free survival were firstly assessed, which indicated significantly worse survival in patients with PHG and corresponded with the stratified analysis of PHG severity. This finding is consistent with that of two previous studies $(24,25)$. However, the prognosis of the mild and severe PHG groups were similar $(p=0.398)$, which was likely due to the relatively small sample size of the latter. In agreement with a previous study (25), PHG showed no predictive value for HCC incidence, which may be attributed to the involvement of multiple oncogenic factors (26).

The MELD score, Child-Pugh class and indicators of portal vein pressure such as portal/splenic vein diameter, thrombosis, and spleen size, were relatively worse in patients with PHG, which is consistent with previous reports $(5,24,27)$. Therefore, PHG was found to be a potential indicator of advanced hepatic disease and poor prognosis in LC patients. In this study, 63\% of the cirrhotic patients presented with $\mathrm{PHG}$, which is similar to the results of previous reports (27-29). Age, hemoglobin content, platelet count and Child-Pugh class were identified as independent risk factors for PHG. Lower hemoglobin content and platelet count, younger age and greater severity of liver disease were associated with an increased risk of PHG, which is consistent with the results of previous studies $(1,5,24)$. Subsequently, we developed an effective risk prediction tool to identify patients at risk of PHG, which showed high sensitivity, PPV, NPV, and accuracy. The non-invasive clinical predictors PSR and RLAR were similarly evaluated in a recent study, and showed an accuracy of 74.7 and $35.1 \%$, and relatively low levels of specificity and NPVs (30). In contrast, our nomogram showed satisfactory discrimination, calibration and clinical utility value. As far as we know, our study is also the first report to assess the diagnostic accuracy of APRI, FIB-4, and GUCI to detect PHG. Interestingly, all the AUC values of APRI, FIB-4, GUCI, Child-Pugh class and MELD score were not satisfactory. This may be due to patient heterogeneity caused by various cirrhosis etiologies.

It should be pointed out that our study has several limitations. First of all, as a retrospective study, the associated certainty of patient information is limited. Second, this study was conducted on the inpatients at a single center, which may not be representative of all cirrhotic patients. Furthermore, factors that may potentially affect PHG, such as blood ammonia level, were not included in the risk factor analysis (28). Therefore, the generalizability of the study findings needs to be further validated through a prospective multicenter study conducted on a larger cohort of patients. 
In conclusion, we developed and validated a visualized, clinical parameter-based nomogram which is a reliable and noninvasive method to predict PHG in cirrhotic patients. Besides, we also explored the relationship between PHG and other decompensated endpoints of LC such as HCC incidence. PHG is an independent risk factor for OS of LC, but not for the occurrence of HCC. PHG and HCC might be independent decompensated endpoint events of LC which suggests PHG cannot be used as an indicator for HCC surveillance.

\section{DATA AVAILABILITY STATEMENT}

The original contributions presented in the study are included in the article/supplementary material, further inquiries can be directed to the corresponding author.

\section{ETHICS STATEMENT}

The studies involving human participants were reviewed and approved by the Ethics Committee of Daping Hospital. Written informed consent for participation was not required for this study in accordance with the national legislation and the institutional requirements.

\section{REFERENCES}

1. Gjeorgjievski M, Cappell MS. Portal hypertensive gastropathy: a systematic review of the pathophysiology, clinical presentation, natural history and therapy. World J Hepatol. (2016) 8:231-62. doi: 10.4254/wjh.v8.i4.231

2. Zardi EM, Ghittoni G, Margiotta D, Viera FT, Di Matteo F, Rossi S. Portal hypertensive gastropathy in cirrhotics without varices: a case-control study. Eur J Gastroenterol Hepatol. (2015) 27:91-6. doi: 10.1097/MEG.0000000000000234

3. Ibrahim M, Degré D, Devière J. Active bleeding caused by portal hypertensive gastropathy. Gastrointest Endosc. (2014) 80:724. doi: 10.1016/j.gie.2014.01.020

4. Primignani M, Carpinelli L, Preatoni P, Battaglia G, Carta A, Prada A, et al. Natural history of portal hypertensive gastropathy in patients with liver cirrhosis. The New Italian Endoscopic Club for the study and treatment of esophageal varices (NIEC). Gastroenterology. (2000) 119:1817. doi: 10.1053 /gast. 2000.8555

5. Fontana RJ, Sanyal AJ, Mehta S, Doherty MC, Neuschwander-Tetri BA, Everson GT, et al. HALT-C Trial Group. Portal hypertensive gastropathy in chronic hepatitis $\mathrm{C}$ patients with bridging fibrosis and compensated cirrhosis: results from the HALT-C trial. Am J Gastroenterol. (2006) 101:98392. doi: 10.1111/j.1572-0241.2006.00461.x

6. Min YW, Bae SY, Gwak GY, Paik YH, Choi MS, Lee JH, et al. A clinical predictor of varices and portal hypertensive gastropathy in patients with chronic liver disease. Clin Mol Hepatol. (2012) 18:17884. doi: $10.3350 / \mathrm{cmh} .2012 .18 .2 .178$

7. Garcia-Tsao G, Sanyal AJ, Grace ND, Carey W, Practice Guidelines Committee of the American Association for the Study of Liver D and Practice Parameters Committee of the American College of Gastroenterology. Prevention and management of gastroesophageal varices and variceal hemorrhage in cirrhosis. Hepatology. (2007) 46:922-38. doi: 10.1002/hep.21907

8. Zardi EM, Uwechie V, Caccavo D, Pellegrino NM, Cacciapaglia F, Di Matteo F, et al. Portosystemic shunts in a large cohort of patients with liver cirrhosis: detection rate and clinical relevance. J Gastroenterol. (2009) 44:76-83. doi: 10.1007/s00535-008-2279-1

9. Elalfy H, Elsherbiny W, Abdel Rahman A, Elhammady D, Shaltout SW, Elsamanoudy AZ, et al. Diagnostic non-invasive model of large risky

\section{AUTHOR CONTRIBUTIONS}

WW and ZM prepared the manuscript. LW and DC designed and drafted the manuscript. WW, ZM, GZ, TW, SL, YG, and $\mathrm{XY}$ were involved in data curation and investigation. WW, ZM, GZ, LW, and DC contributed to the interpretation of the results. TW, SL, YG, and XY were responsible for data analyzing and visualization. All authors contributed to the article and approved the submitted version.

\section{FUNDING}

This work was supported by National Natural Science Foundation of China (NCFS:81802459), Chongqing Natural Science Foundation (cstc2018jcyjAX0603), and Sponsored by Science and Technology Innovation Enhancement Project of Army Medical University (2019XLC3045).

\section{ACKNOWLEDGMENTS}

The authors are grateful for all endoscopists in Daping Hospital, the Third Affiliated Hospital of Army Medical University, China.

esophageal varices in cirrhotic hepatitis C virus patients. World J Hepatol. (2016) 8:1028-37. doi: 10.4254/wjh.v8.i24.1028

10. Petzold G, Tsaknakis B, Bremer S, Knoop RF, G GR, Amanzada A, et al. Evaluation of liver stiffness by 2D-SWE in combination with noninvasive parameters as predictors for esophageal varices in patients with advanced chronic liver disease. Scand J Gastroentero. (2019) 54:3429. doi: 10.1080/00365521.2019.1585571

11. McCormack TT, Sims J, Eyre-Brook I, Kennedy H, Goepel J, Johnson AG, et al. Gastric lesions in portal hypertension: inflammatory gastritis or congestive gastropathy? Gut. (1985) 26:1226-32. doi: 10.1136/gut.26.11. 1226

12. Andres-Otero MJ, De-Blas-Giral I, Puente-Lanzarote JJ, SerranoAullo T, Morandeira MJ, Lorente S, et al. Multiple approaches to assess fourteen non-invasive serum indexes for the diagnosis of liver fibrosis in chronic hepatitis C patients. Clin Biochem. (2016) 49:560-5. doi: 10.1016/j.clinbiochem.2015.12.017

13. Jiang Y, Tang H, Wang Z, Sun Y, Meng W, Wang G, et al. Two nomograms to select hepatocellular carcinoma patients with macroscopic vascular invasion for hepatic resection. J Cancer. (2018) 9:3287-94. doi: 10.7150/jca. 25899

14. Steyerberg EW, Harrell FJ, Borsboom GJ, Eijkemans MJ, Vergouwe Y, Habbema JD. Internal validation of predictive models: efficiency of some procedures for logistic regression analysis. J Clin Epidemiol. (2001) 54:77481. doi: 10.1016/S0895-4356(01)00341-9

15. Steyerberg EW, Bleeker SE, Moll HA, Grobbee DE, Moons KG. Internal and external validation of predictive models: a simulation study of bias and precision in small samples. J Clin Epidemiol. (2003) 56:4417. doi: 10.1016/S0895-4356(03)00047-7

16. Harrell FE, Lee KL, Califf RM, Pryor DB, Rosati RA. Regression modelling strategies for improved prognostic prediction. Stat Med. (1984) 3:14352. doi: 10.1002/sim.4780030207

17. Harrell FE, Califf RM, Pryor DB, Lee KL, Rosati RA. Evaluating the yield of medical tests. JAMA. (1982) 247:25436. doi: 10.1001/jama.1982.03320430047030

18. Steyerberg EW, Vergouwe Y. Towards better clinical prediction models: seven steps for development and an ABCD for validation. Eur Heart J. (2014) 35:1925-31. doi: 10.1093/eurheartj/ehu207 
19. Holmberg L, Vickers A. Evaluation of prediction models for decisionmaking: beyond calibration and discrimination. PLoS Med. (2013) 10:e1001491. doi: 10.1371/journal.pmed.1001491

20. Vickers AJ, Cronin AM, Elkin EB, Gonen M. Extensions to decision curve analysis, a novel method for evaluating diagnostic tests, prediction models and molecular markers. BMC Med Inform Decis Mak. (2008) 8:53. doi: 10.1186/1472-6947-8-53

21. Li Q, Lu C, Li W, Huang Y, Chen L. Evaluation of eLIFT for non-invasive assessment of liver fibrosis and cirrhosis in patients with chronic hepatitis B virus infection. Sci Rep. (2017) 7:5429. doi: 10.1038/s41598-017-05718-x

22. Dreiseitl S, Harbauer A, Binder M, Kittler H. Nomographic representation of logistic regression models: A case study using patient self-assessment data. $J$ Biomed Inform. (2005) 38:389-94. doi: 10.1016/j.jbi.2005.02.006

23. Shariat SF, Capitanio U, Jeldres C, Karakiewicz PI. Can nomograms be superior to other prediction tools? BJU Int. (2009) 103:4925. doi: 10.1111/j.1464-410X.2008.08073.x

24. Kim MY, Choi H, Baik SK, Yea CJ, Won CS, Byun JW, et al. Portal hypertensive gastropathy: correlation with portal hypertension and prognosis in cirrhosis. Dig Dis Sci. (2010) 55:3561-7. doi: 10.1007/s10620-0101221-6

25. Bang CS, Kim HS, Suk KT, Kim SE, Park JW, Park SH, et al. Portal hypertensive gastropathy as a prognostic index in patients with liver cirrhosis. Bmc Gastroenterol. (2016) 16:93. doi: 10.1186/s12876-016-0508-2

26. Fujiwara N, Friedman SL, Goossens N, Hoshida Y. Risk factors and prevention of hepatocellular carcinoma in the era of precision medicine. J Hepatol. (2018) 68:526-49. doi: 10.1016/j.jhep.2017.09.016

27. De Lisi S, Peralta S, Arini A, Simone F, Craxi A. Oesophagogastroduodenoscopy in patients with cirrhosis: extending the range of detection beyond portal hypertension. Digest Liver Dis. (2011) 43:48-53. doi: 10.1016/j.dld.2010.04.004
28. El-Kalla F, Mansour L, Kobtan A, Elzeftawy A, Abo AL, AbdElsalam S, et al. Blood ammonia level correlates with severity of cirrhotic portal hypertensive gastropathy. Gastroent Res Pract. (2018) 2018:9067583. doi: 10.1155/2018/9067583

29. Wu Q, Shen L, Chu J, Ma X, Jin B, Meng F, et al. Characterization of uncommon portosystemic collateral circulations in patients with hepatic cirrhosis. Oncol Lett. (2015) 9:347-50. doi: 10.3892/ol.2014.2626

30. Mandhwani R, Hanif FM, Ul Haque MM, Wadhwa RK, Hassan Luck N, Mubarak M. Noninvasive clinical predictors of portal hypertensive gastropathy in patients with liver cirrhosis. J Transl Int Med. (2017) 5:16973. doi: 10.1515/jtim-2017-0025

Conflict of Interest: The authors declare that the research was conducted in the absence of any commercial or financial relationships that could be construed as a potential conflict of interest.

Publisher's Note: All claims expressed in this article are solely those of the authors and do not necessarily represent those of their affiliated organizations, or those of the publisher, the editors and the reviewers. Any product that may be evaluated in this article, or claim that may be made by its manufacturer, is not guaranteed or endorsed by the publisher.

Copyright (C) 2022 Wang, Mu, Zhu, Wang, Lai, Guo, Yin, Wen and Chen. This is an open-access article distributed under the terms of the Creative Commons Attribution License (CC BY). The use, distribution or reproduction in other forums is permitted, provided the original author(s) and the copyright owner(s) are credited and that the original publication in this journal is cited, in accordance with accepted academic practice. No use, distribution or reproduction is permitted which does not comply with these terms. 